\title{
lodoprophylaxis and thyroid autoimmunity: an update
}

\author{
Claudia Teti ${ }^{1} \cdot$ Marta Panciroli $^{2,3} \cdot$ Elena Nazzari $^{4} \cdot$ Giampaola Pesce $^{2,5} \cdot$ Stefano Mariotti $^{6} \cdot$ Antonella Olivieri $^{7}$. \\ Marcello Bagnasco ${ }^{2}$
}

Received: 26 January 2021 / Revised: 6 March 2021 / Published online: 29 April 2021

(c) The Author(s) 2021

\begin{abstract}
Adequate iodine intake is necessary for normal thyroid function. Iodine deficiency is associated with serious complications, but also iodine excess can lead to thyroid dysfunction, and iodine supplementation aimed to prevent iodine deficiency disorders has been associated with development of thyroid autoimmunity. The epidemiology of thyroid diseases has undergone profound changes since the implementation of iodoprophylaxis, notably by means of iodine-enriched salt, specifically resulting in decreased prevalence of goiter and neonatal hypothyroidism, improved cognitive function development in infancy, and reduced incidence of more aggressive forms of thyroid cancer. The main question we address with this review is the clinical relevance of the possible effect on autoimmunity exerted by the use of iodine-enriched salt to correct iodine deficiency. In animal models, exogenous iodine is able to trigger or exacerbate thyroid autoimmunity, but it is still not clear whether the observed immunological changes are due to a direct effect of iodine on immune response, or whether they represent a secondary response to a toxic effect of iodine on thyroid tissue. Previous iodine status of a population seems to influence the functional thyroid response to increased iodine intake and possibly the development of thyroid autoimmunity. Moreover, the prevalence of thyroid antibodies, regarded as hallmark of autoimmune thyroid disease, varies between populations under the influence of genetic and environmental factors, and the presence of thyroid antibodies does not always coincide with the presence of thyroid disease or its future development. In addition, the incidence of autoimmune diseases shows a general increasing trend in the last decades. For all these reasons, available data are quite heterogeneous and difficult to analyze and compare. In conclusion, available data from long-term population surveys show that a higher than adequate population iodine intake due to a poorly controlled program of iodine prophylaxis could induce thyroid dysfunction, including thyroid autoimmunity mostly represented by euthyroid or subclinical hypothyroid autoimmune thyroiditis. Close monitoring iodine prophylaxis is therefore advised to ensure that effects of both iodine deficiency and iodine excess are avoided.
\end{abstract}

Keywords Thyroid $\cdot$ Thyroid autoimmunity $\cdot$ Iodine $\cdot$ Iodized salt $\cdot$ Goiter

Claudia Teti and Marta Panciroli contributed equally to this work.

Marcello Bagnasco

bagnasco@unige.it

Endocrinology and Diabetology Unit, ASL 1, Imperia, Italy

2 Department of Internal Medicine and Medical Specialties (DiMI), Genoa University, Viale Benedetto XV, 6 I-16132 Genova, Italy

3 Department of Neurosciences, Rehabilitation, Ophthalmology, Genetics, Maternal and Child Health, University of Genoa, Genoa, Italy
4 Endocrinology and Diabetology Unit, ASL 2, Savona, Italy

5 Laboratory of Autoimmunology, IRCCS Ospedale Policlinico San Martino, Genova, Italy

6 Department of Medicine and Public Health, University of Cagliari, Cagliari, Italy

7 Department of Cardiovascular and Endocrine-Metabolic Diseases and Aging, Italian National Institute of Health, Viale Regina Elena, 299, 00161 Roma, Italy 


\section{Introduction: thyroid autoimmunity and iodine}

Autoimmune thyroid diseases (AITD) are the most common autoimmune disorders [1,2]. Autoimmune thyroiditis (AT) is the paradigm of "destructive" organ-specific autoimmunity, and Graves' disease (GD) is the unique clinically relevant case of organ-specific autoimmune disease resulting in hyperfunction of the target organ and the most common cause of hyperthyroidism with the possible exception of areas with severe iodine deficiency. The multi-faceted clinical presentation is due to multiple pathogenetic mechanisms involving (cell-mediated in autoimmune hypothyroidism due to thyroid destruction and autoantibody-mediated in autoimmune hyperthyroidism) [3-5]. In any case, the presence of thyroid autoantibodies has been regarded for decades as a hallmark of AITD: notably, antibodies directed against thyroid peroxidase (TPOAb) or thyroglobulin $(\mathrm{TgAb})$ [6]. Such autoantibodies are present in both AT and GD, although more frequently and at higher levels in the former. The prevalence of thyroid antibodies varies between populations under the influence of genetic [7-10] and environmental $[7,11]$ factors.

The prevalence of AT is far higher than that of GD (about 5- to tenfold) [1]. Therefore, the presence of such autoantibodies is predominantly associated with decreased thyroid function. It has to be underlined that the presence of circulating TPOAb or TgAb does not always coincide with the presence of disease: clinical disease requires significant organ damage, in which cell-mediated mechanisms play a major role, as above mentioned. Such thyroid autoantibodies can be predictive of disease development over time [12] but also be a transient phenomenon; thus, their prevalence is higher than clinical disease [13]. On the other hand, TSHreceptor autoantibodies (TRAb) are the only autoantibodies displaying a direct pathogenetic role, most often causing hyperthyroidism due to their thyroid-stimulating activity: in fact, since the development of highly sensitive assays [14], their prevalence is closely similar to that of clinical GD.
Normal thyroid activity is dependent on iodine intake. In fact, iodine is the most important environmental factor in upholding thyroid function [15].

Iodine deficiency has been since centuries a major epidemiological problem. In fact, since the very beginning of life outside the oceans, some 400 million years ago, the availability of iodine in the environment became very poor: there is circumstantial very early evidence for iodine deficiency disorders in Homo sapiens as well as in Neanderthals, and iodine availability and the ability to use it has probably been an important factor in human evolution [16]. Endemic goiter is the most visible sign of iodine deficiency, but its most devastating consequence is brain damage causing mental retardation in children.

In Table 1, the optimal levels of iodine intake by age and population group according to the World Health Organization (WHO) and the United States Institute of Medicine (IOM) are reported. Iodine supplementation through salt iodization, implemented by the WHO and the International Council for the Control of Iodine Deficiency Disorders (ICCIDD, now Iodine Global Network), is a worldwide, effective strategy for preventing iodine deficiency-related problems. Its safety and efficacy profile has been extensively investigated, and benefits far outweigh the potential iodineinduced risks $[17,18]$. Iodine prophylaxis through iodized salt has been shown to modulate the pattern of thyroid diseases and also to exert a pivotal role in abating iodine deficiency disorders (IDD) [19, 20].

Iodine excess also exerts important effects on thyroid function, being able to transiently block it (Wolff-Chaikoff effect), or producing long-lasting hypothyroidism; moreover, iodine excess may cause transient destructive thyrotoxicosis via toxic damage, or sustained hyperthyroidism in the presence of autonomously functioning tissue [21]. Finally, and more relevant to this review, thyroid autoimmunity can be driven by exposure to iodine excess.

For years, several mechanisms have been suggested to explain the immunomodulatory effects of iodine and specifically the relationship between the level of iodine intake and
Table 1 Recommendations for iodine intake $(\mu \mathrm{g} / \mathrm{d})$ by age or population group

\begin{tabular}{lllll}
\hline & IOM & & WHO \\
\hline Age or population group & EAR & AI or RDA & Age or population group & RNI \\
Infants 0-12 months & & $110-130$ & Children 0-5 yr & 90 \\
Children 1-8 yr & 65 & 90 & Children 6-12 yr & 120 \\
Children 9-13 yr & 73 & 120 & & \\
Adults $\geq 14$ yr & 95 & 150 & Adults $\geq 12$ yr & 150 \\
Pregnancy & 160 & 220 & Pregnancy & 250 \\
Lactation & 200 & 290 & Lactation & 250 \\
\hline
\end{tabular}

$I O M$, United States Institute of Medicine; $E A R$, estimated average requirement; $A I$, adequate intake; RDA, recommended daily allowance; $R N I$, recommended nutrient intake; WHO, World Health Organization 
thyroid autoimmunity $[22,23]$. It is still not clear whether the observed immunological changes are due to a direct iodine effect on immune effector cells, or whether they represent a secondary response to a metabolic and/or toxic effect of iodine on thyroid tissue. It has been hypothesized that a sudden shift from very low to high iodine intake may induce damage to thyroid tissue by free radicals [24, 25] whose importance in triggering/exacerbating thyroid autoimmunity is presently widely recognized [26-28].

The role of iodine in inducing thyroid autoimmunity is strongly supported by animal models. Excess of iodine can anticipate and exacerbate the occurrence of spontaneous thyroiditis in genetically predisposed animals, by increasing the immunogenicity of thyroglobulin $(\mathrm{Tg})$ [23, 29-31]. Thyroglobulin is an iodinated protein and is an integral part of the hormone synthesizing mechanisms of the thyroid follicle.

Some authors described iodinated thyroglobulin eliciting a greater immune response compared with less iodinated thyroglobulin molecules in in vitro experiments $[29,32,33]$. It has been demonstrated that the enhanced iodination of thyroglobulin induces the expression of new cryptic epitopes on the molecule that could be responsible for triggering the autoimmune process [30]. The rise of $\mathrm{TgAb}$ associated with iodine intake in humans, similarly to what is observed in animals, is likely due to an increased immunogenicity of $\mathrm{Tg}[30,34-36]$. This may be explained by the fact that $\mathrm{Tg}$ is the only self-antigen that undergoes post-translational modification as a consequence of the environmental supply of iodine, with the exposure of previously hidden epitopes. Direct evidence supporting the latter mechanism was recently provided by Latrofa et al. [37] who carried out a detailed study of the fine specificity of $\mathrm{TgAb}$ detected in sera of an endemic population before and after implementation of iodine prophylaxis.

However, while the role of the iodine intake level in the etiology of non-autoimmune thyroid disorders is well established, the association between population iodine intake level and AITD is more devious [6]. Taking into account the global health benefit of iodine prophylaxis implementation, the question arises as to whether the implementation of universal salt iodization has led to an increased incidence of AITD and, if so, what is its clinical relevance/impact.

\section{Does iodoprophylaxis induce thyroid autoimmunity?}

As above mentioned, it is well known that variable iodine intake may be accompanied by thyroid disease: adverse consequences of low iodine intake are a major epidemiological problem, but thyroid dysfunction may also occur at higher intake levels [38-40].
In subjects with an underlying thyroid disorder (underlying autoimmune thyroiditis, previous treatment with radioactive iodine or history of external thyroid irradiation, previous subtotal thyroidectomy, postpartum or subacute thyroiditis and intake of some medications, such as lithium, which interferes with iodine organification and thyroid hormones release), acute excessive iodine intakes may lead to temporary overt or subclinical hypothyroidism that resolves when iodine intakes decrease [41].

Moreover, many studies indicate that even relatively small changes in the level of iodine intake of a population may result in changes in the spectrum of thyroid diseases [42]. Iodine-induced hyperthyroidism has been observed at daily iodine intakes of less than $300 \mu \mathrm{g}[43,44]$. An increased incidence of hyperthyroidism in previously iodine-deficient areas has been described, although transiently, even during careful implementation of universal salt iodization [15, 45-47]. Therefore, the World Health Organization (WHO) recommends that iodine fortification of a population should be initiated cautiously and be followed by a monitoring program to register its effects and counteract any side events.

It has been proposed the term "iodine memory" [15] when evaluating the association between current iodine intake and the epidemiology of thyroid disease in a population, because the degree of a previous exposure to different level of iodine intake (low or high) may have influenced the current occurrences of diseases. An increase from low to normal iodine intake is associated with a reduction in thyroid size in the population within a few years [48], while thyroid nodularity is more or less irreversible [49]. It remains to be elucidated if the high frequency of hypothyroidism in areas with excessive iodine intake is a reversible phenomenon. Studies of hypothyroid patients living in Japan and Korea with high iodine intake have indicated that a reduction in iodine intake may normalize thyroid function in some of these patients [50, 51]. Individuals with preexisting thyroid disease or those previously exposed to iodine deficiency may be more susceptible to thyroid disorders due to an increase in iodine intake, in some cases at intakes only slightly above physiological needs. Thyroid dysfunction due to excess iodine intake is usually mild and transient, but iodine-induced hyperthyroidism can be life-threatening in some individuals [21].

The question arises as to such "side pathological effects" are related to the development of thyroid autoimmunity, although there is no doubt that other factors play a role (specifically, the development of hyperthyroidism in iodinedeficient areas where the prevalence of nodular goiter, and consequently the likelihood of nodular functional autonomy, is high).

The effect of public iodization programs on the development of thyroid antibodies is yet uncertain. As previously described, high iodine intake may trigger thyroid 
autoimmunity, and it might be expected that the prevalence of harboring circulating thyroid antibodies would be low in populations with a low iodine intake and high in populations with a high iodine intake. However, the association between iodine intake and the presence of circulating thyroid antibodies is considerably more complex, taking also into consideration that multiple genetic and environmental factors are involved in the pathogenesis of autoimmune thyroiditis.

The most common autoantibodies against the thyroid gland associated with autoimmune thyroiditis are thyroid peroxidase antibody (TPOAb) and thyroglobulin antibody ( $\mathrm{TgAb}$ [ [52]. Antibody levels seem to correlate well with the severity of histological thyroiditis [53], but in some cases, histological evidence of autoimmune thyroiditis is not accompanied by detectable TPOAb or TgAb in blood [54].

As already noted, it is difficult to interpret and compare the many studies where both thyroid antibodies and iodine status have been measured in one or more populations [55].

Iodine deficiency has been associated with goiter and people with nodular goiter relatively often have circulating thyroid antibodies [56, 57], probably caused by enhanced release of thyroid antigens from the abnormal gland. Thus, circulating thyroid antibodies are probably more common in populations where goiter is common because of iodine deficiency. Consistently with such a scenario, recent findings indicate that iodine deficiency in early pregnancy is associated with higher risk of TPOAb and $\mathrm{TgAb}$ positivity and that the presence of both autoantibodies at high titers is associated with increased risk of subclinical or overt hypothyroidism [58], and optimal iodine supply results in the lowest risk for thyroid autoantibody positivity [59].

It has also been reported that the prevalence of lymphocytic thyroid infiltration becomes high after an increase in population iodine intake [60]. Moreover, a sudden increase in iodine intake in an iodine-deficient population may induce enhanced thyroid autoimmunity [61], but this may be a transient phenomenon [62].

Two groups of Sri Lankan schoolgirls were studied in 1998 and 2001 in relation to the evolution of thyroid autoimmunity, with the change in goiter prevalence, during 3 years of iodine prophylaxis [63]. This study demonstrates that in 2001, goiter prevalence and thyroid autoimmunity rates were significantly lower than in 1998; the pattern of thyroid $\mathrm{Ab}$ was different in the two groups (more TPOAb in 2001 group); in 2001 alone, the occurrence of hypothyroidism was correlated with the presence of thyroid autoimmunity. These results indicated, for the first time, an evolution of thyroid autoimmunity markers during the course of iodine prophylaxis: when iodine supplementation is first introduced it may have induced the greater prevalence of $\mathrm{TgAb}$ in 1998 in comparison with 2001. It is noteworthy that the reversibility of thyroid autoimmunity occurred mainly in those individuals who showed low titers of $\mathrm{TgAb}$ alone in 1998: this fact suggests that the immune system's response to iodine intake could be heterogeneous, possibly reflecting different immunological background.

In Greece, thyroid autoimmunity has been positively associated with increased iodine intake, as well as with the female gender [64]. Moreover, mean TSH values were increased in females and decreased with age. The latter is probably due to the presence of autonomous goiter in older subjects, as a result of long-term status of iodine deficiency in the past.

As well, an increased prevalence of thyroid autoantibody positivity and subclinical hypothyroidism was observed in Denmark, after 4-5 years from the implementation of a cautious iodoprophylaxis program in 3570 members from the original cohort [65]. Similar results were obtained in the Pescopagano's survey, a small rural community followed up for as long as 15 years from iodoprophylaxis with iodized salt implementation, which in turn resulted in marked reduction of the prevalence of goiter and thyroid autonomy [66].

However, other studies report no increase or a reduction in thyroid autoimmunity with time following iodine salt fortification. A large (more than 350,000 people) retrospective study conducted in Tasmania between 1995 (median population UIC: $75 \mu \mathrm{g} / \mathrm{L}$ ) and 2013 (median population UIC: $108 \mu \mathrm{g} / \mathrm{L}$ ) and aimed at analyzing the relationship between trends in TSH and TPOAb testing and different phases of iodine nutrition failed to detect any increasing trend over time of TPOAb following iodine salt fortification, whereas a decreasing trend of overt thyroid dysfunction was apparent [67]. Another study, which analyzed the impact of iodine nutrition on thyroid diseases few years after the introduction of the iodine prophylaxis program (1997-2001, median population UIC: $123 \mu \mathrm{g} / \mathrm{L}$ ) and more recently in Northeast Germany (2008-2012, median population UIC: $112 \mu \mathrm{g} / \mathrm{L}$ ), also found a stable prevalence of markers of thyroid autoimmune disorders and a decreased prevalence of goiter [68]. More recently, in a study conducted in China after two decades of universal salt iodination program, a stable prevalence of thyroid antibody positivity in the population has been reported. This study also found an inverse relationship between iodine intake and thyroid antibodies, suggesting that UIC between 100 and $300 \mu \mathrm{g} / \mathrm{L}$ is optimal and safe for thyroid autoimmunity [69]. More specifically, a number of data suggest that only iodine supplementation regimens attaining relatively high median UIC levels (i.e., $200 \mu \mathrm{g} / \mathrm{L}$ or more) are able to induce relevant long-term alteration of thyroid function due to autoimmune disease, and the relationship between population iodine supply and occurrence of AITD could be depicted by a "U-shaped" function [70, 71]: in other words, the likelihood of developing thyroid autoimmune diseases would increase when median population UIC exceeds approximately $300 \mu \mathrm{g} / \mathrm{L}$, but as well 
when is less than $100 \mu \mathrm{g} / \mathrm{L}$ (see Fig. 1). The majority of these results come from Chinese populations, in whom marked differences in iodine supply in single region have been observed due to different iodine supply strategies [69, 71-75]. In line with such a scenario, according to at least two recent reports cited above $[58,59]$ both iodine deficiency and iodine excess are associated with increased risk of thyroid autoantibody positivity in pregnancy.

Moreover, circulating thyroid antibodies are not extraordinarily common in populations with stable high iodine intake [38], although thyroid dysfunction is more frequent when the iodine intake is high [76].

Importantly, most recent data from the Danish survey in North Jutland, involving an open cohort of over 300,000 individuals, demonstrated no increase of overt hypothyroidism (related, as a rule, to autoimmune thyroiditis) and a decrease of hyperthyroidism (due to thyroid autonomy, but also due to autoimmunity, i.e., Graves' disease) over a period of more than 15 years following implementation of mandatory iodine fortification [77].

Finally, and on the same line of evidence, the last data derived from the Sri Lanka iodoprophylaxis survey showed that more than 20 years after its implementation the prevalence of $\mathrm{TgAb}$ consistently declined, and no increase of the prevalence of hypothyroidism (even subclinical) was recorded [78].

Thus, although several reports suggest a somehow promoting effect of iodine salt fortification on thyroid autoimmunity, its role in causing clinically relevant autoimmune thyroid disease in long term is not clearly proven.

\section{Discussion}

As described above, the epidemiology of thyroid diseases has undergone profound changes since the implementation of iodoprophylaxis, specifically resulting in decrease of the prevalence of goiter and neonatal hypothyroidism, as well as in improved cognitive function development in infancy and reduced incidence of more aggressive forms of thyroid neoplasia. Thus, there is no doubt about the benefits of iodoprophylaxis for the general population and specifically for newborns, children, and pregnant women. The main question we have intended to address with the present review is the following: is an increase of thyroid autoimmunity a price to pay for such benefits, or the risk of a clinically significant increase of autoimmune disease is neglectable?

Undoubtedly, it is quite difficult to provide a definite evidence-based answer to the main question (does iodoprophylaxis increase the prevalence of clinically significant thyroid dysfunction due to thyroid autoimmunity?) for a number of reasons:

- Definition of clinically apparent thyroid autoimmune disease or simply autoantibody prevalence: as generally recognized, autoantibody positivity, especially for TPOAb, confers a risk of, but not necessarily results in progression to clinical disease.

- Possible transient effects: autoantibody positivity, especially at low titers, and subclinical hypothyroidism (i.e., slight increase of TSH with Thyroxine concentrations within the normal range) may be transient.
Fig. 1 Summary of expected effects of iodine deficiency/ excess and iodoprophylaxis. Reference numbers are the same as in the main text. UIC: urinary iodine concentration

\section{IODOPROPHYLAXIS}

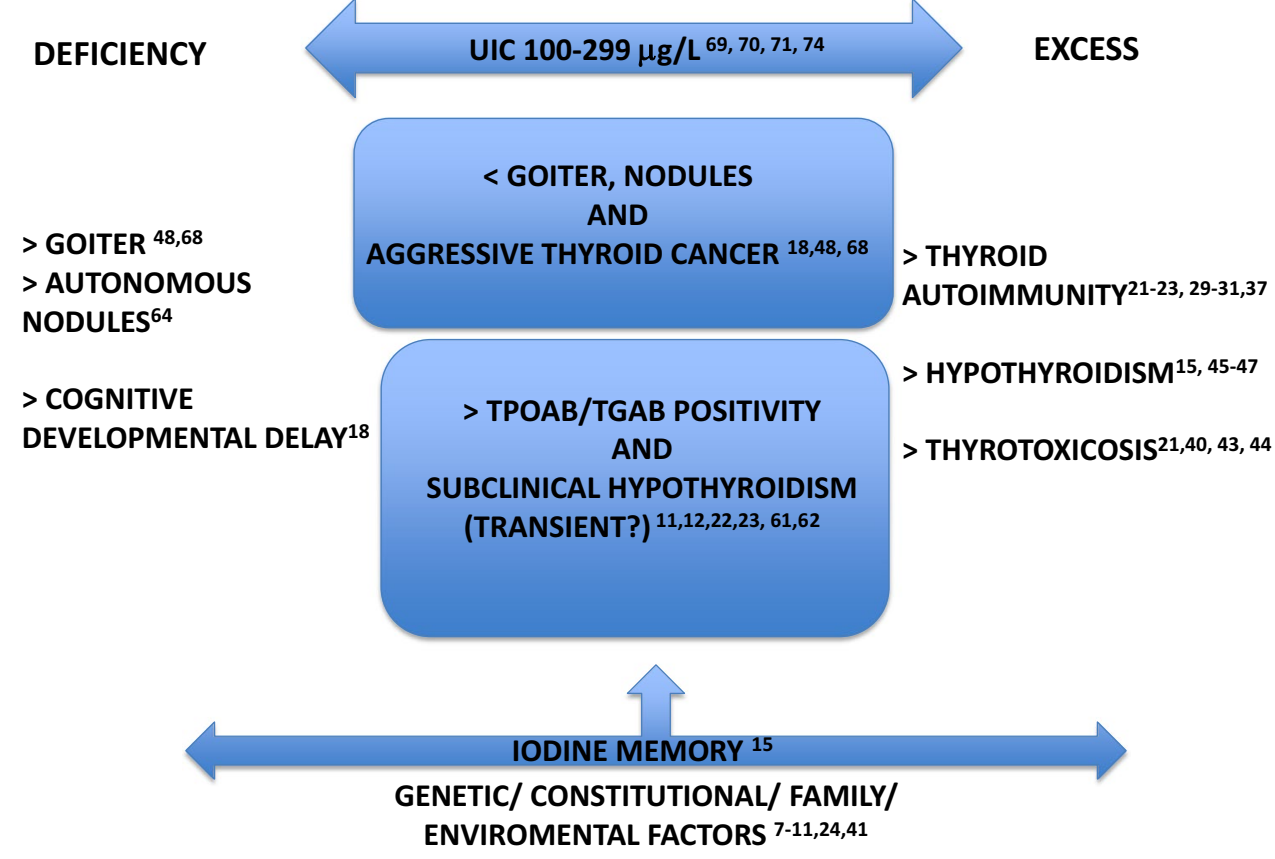


- Possible effect of iodine status (mild or moderate/severe iodine deficiency) before iodoprophylaxis implementation.

- Evolution of autoimmune disease epidemiology independently of iodine supply.

As far as the first two points are concerned, it has to be noted that the numerous published studies, cited in the previous section, are quite heterogeneous, so that a meta-analysis, or also a rigorous systematic review, is hardly feasible. Salt iodization has been implemented and promoted in different ways, either mandatory or on a voluntary basis, in different ethnic groups, at different levels of baseline iodine intake. Most studies are cross-sectional, in some cases evaluating individuals of the same ethnic group living in areas with different iodine intake, or were repeated years (up to two decades) after prophylaxis implementation: numbers of evaluated individuals ranged from hundreds to tens/hundreds of thousands.

In addition, TPOAb and thyroid function have been evaluated in the majority of studies. As described above, an increase of thyroid autoantibodies (TPOAb and/or $\mathrm{TgAb}$ ) following implementation of prophylaxis by means of iodized salt, and an increased prevalence of subclinical hypothyroidism, has been consistently reported. However, in a significant proportion of patients, subclinical hypothyroidism may reverse to full euthyroidism [79]. Again, it is worth noting that in a number of studies, no significant increase of thyroid autoimmunity was observed when iodine intake was adequate.

Most often, published studies refer to autoantibody (especially TPOAb) positivity as an indicator of the presence of, or risk to develop thyroid autoimmune disease, with consequent thyroid dysfunction (more often, subclinical hypothyroidism). However, a reliable indicator of structural thyroid damage due to autoimmune thyroiditis is thyroid ultrasonography, whose specificity and sensitivity under this respect have been underlined [80, 81]. Only few data, to our knowledge, have been published concerning the prevalence of hypoechoic pattern, typical of autoimmune thyroiditis following implementation of iodoprophylaxis with iodized salt: Miranda et al., in Brazil, reported an increase of hypoechoic pattern in children following attainment of high $(300 \mu \mathrm{g} / \mathrm{L}$ or more $)$ UIC concentration, and a trend to decrease when the target was adjusted at $165 \mu \mathrm{g} / \mathrm{L}$ [73]. In addition, in a recent study reporting data after a decade of iodine prophylaxis on voluntary basis in Italy [82], the frequency of moderately or markedly hypoechoic patterns in schoolchildren residing in iodine-sufficient areas (median UIC value $129 \mu \mathrm{g} / \mathrm{L}$ ) was $6.6 \%$, whereas it was significantly higher $(10.9 \%)$ in an area of Sicily region where UIC value was still under the desired target value (median UIC value $89 \mu \mathrm{g} / \mathrm{L}$ ). Even more importantly, in Liguria, one of the studied Italian regions where comparative dataset collected 8 years before (i.e., 2 years following iodized salt consumption implementation) were available, no increase in the frequency of hypoechoic pattern was observed $(3.3 \%$ at $2,2.7 \%$ at 10 years) despite a significant reduction in goiter prevalence. These data suggest that iodoprophylaxis aimed to attain an UIC target indicative of an adequate iodine intake does not result in a structural damage of the thyroid typical of autoimmune thyroiditis.

Concerning the third point, it has been suggested that the possible inducing effect of iodine supply on thyroid autoimmunity is at least in part related to baseline iodine status, namely, the higher the degree of iodine deficiency, the higher the likelihood of autoimmunity induction.

That iodine supply in conditions of severe iodine deficiency may cause thyroid dysfunction is a well-known fact, but it is mainly related to the development of hyperthyroidism due to thyroid autonomy in nodular goiters [43]: nowadays, however, due to the present iodine nutritional status in the majority of countries [19], the risk of toxicity of autonomously functioning thyroid nodules induced by sudden iodine repletion is probably minimal. On the other hand, data on the relationship between baseline iodine status and autoimmunity development are at least in part contradictory. In any case, considering the global amelioration of iodine nutrition worldwide and the reduction of impact of moderate/severe iodine deficiency, the relevance of this point is probably minor.

Concerning the fourth point, namely, the general evolution of autoimmune disease epidemiology, there is little doubt that the prevalence of autoimmune diseases has progressively increased during the last decades [83, 84], with an inverse correlation with infectious disease epidemiology and a direct correlation with "well-being" indicators (income, improved hygienic conditions), in both developed and developing countries [85]. More than 10 years ago, proof of such a relationship was specifically provided for thyroid autoimmunity [86]. This implies that studies evaluating the increase in thyroid autoimmune diseases following iodoprophylaxis implementation over a decade or more may be affected by the bias of the general trend to increase in autoimmune phenomena per se. Such a trend is hardly quantifiable but also may be hardly ruled out.

In summary, literature data are at least in part controversial, as previously reported in detail. However, on some points, there is substantial agreement:

- Iodine excess (UIC > $300 \mu \mathrm{g} / \mathrm{L}$ ) (see Table 2) may result in altered thyroid function, and this is at least in part due to the development of thyroid autoimmunity. The molecular mechanisms whereby iodine affects the development of thyroid autoimmunity are known or conceivable. 
Table 2 Maximum recommended daily intake levels of iodine and corresponding upper urinary iodine concentration (UIC) thresholds for iodine excess ( modified from ref [21])

\begin{tabular}{lllll}
\hline & $\mathrm{IOM}(\mu \mathrm{g} / \mathrm{die})$ & $\mathrm{SCF}(\mu \mathrm{g} / \mathrm{die})$ & $\begin{array}{l}\mathrm{WHO}(\mu \mathrm{g} / \\
\mathrm{kg} / \mathrm{die})\end{array}$ & $\begin{array}{l}\mathrm{UIC} \\
(\mu \mathrm{g} / \mathrm{L})\end{array}$ \\
\hline $7-10 \mathrm{yr}$ & $\begin{array}{c}600(9-13 \\
\mathrm{aa})\end{array}$ & 300 & $50(7-12 \mathrm{aa})$ & 300 \\
& 1100 & 600 & & $\mathrm{ND}$ \\
Adults & 1100 & 600 & 40 & 500 \\
$\begin{array}{l}\text { Pregnant \& } \\
\text { lactating } \\
\text { women }\end{array}$ & & & & \\
\hline
\end{tabular}

$I O M$, United States Institute of Medicine; $S C F$, The European Union Scientific Committee on Foods; WHO, World Health Organization; $U I C$, urinary iodine concentration; $N D$, not determined

- Implementation of a not adequately monitored iodine prophylaxis may result in increased evidence of "autoimmune phenomena" concerning the thyroid gland.

- The clinical impact of such phenomena (either transient or evolving in clinically apparent thyroid dysfunction) mainly depends upon the target level of iodine supply attained.

As a matter of fact, consistent data support the concept that implementation of iodoprophylaxis programs aimed to reach iodine sufficiency and at the same time to avoid iodine excess (i.e., maintaining population UIC within a relatively narrow interval, as in many European countries, including Italy) does not definitely result in long-term increased incidence of clinically relevant autoimmune thyroid disease (see Fig. 1). Under this respect, the last data from Danish and Sri Lanka surveys showing no increase of hypothyroidism incidence and a decrease of hyperthyroidism due to Graves' disease about two decades after starting iodoprophylaxis program are of particular interest, as well as the results of recent studies on Chinese populations [69, 74, 77]. As mentioned above, a "U-shaped" relationship between iodine intake and the risk of developing thyroid autoimmune disease is conceivable, namely, both iodine excess (even moderate) and also iodine deficiency may increase such risk [71].

\section{Conclusion}

We can conclude that an increased incidence of "autoimmune thyroid phenomena" following implementation of iodoprophylaxis by means of iodine-enriched salt consumption on a voluntary basis is likely; however, its long-term clinical relevance is probably scarce. Nonetheless, a population iodine supply higher than adequate due to a poorly controlled iodoprophylaxis could induce clinically relevant thyroid dysfunction. Therefore, monitoring iodoprophylaxis with standardized population surveys is mandatory, to ensure that both iodine deficiency and iodine excess are avoided. In this regard and on the basis of the experience from Italy and other countries [20,69], we are confident that the routine assessment of thyroid hypoechogenicity in monitoring iodine prophylaxis programs in populations will provide the advantage of easily and quickly identifying even minimal modifications of this frequency over time and to allow the appropriate adjustments of the iodine prophylaxis program if necessary.

Finally, since studies showing a lack of increased thyroid autoimmunity after implementation of universal salt iodization program have reported median UIC values ranging $100-199 \mu \mathrm{g} / \mathrm{L}$ if conducted in children [62] and $100-299 \mu \mathrm{g} / \mathrm{L}$ if conducted in adults [70], we acknowledge the need for further studies aimed at evaluating the possible advantage of using narrow age-related UIC intervals upon which iodine sufficiency is achieved and increase in the baseline frequency of thyroid autoimmunity is avoided at all classes of age.

Abbreviations AITD: Autoimmune thyroid diseases; AT: Autoimmune thyroiditis; GD: Graves' disease; TPOAb: Thyroid peroxidase antibodies; TgAb: Thyroglobulin antibodies; Tg: Thyroglobulin; TRAb: TSHreceptor autoantibodies; IDD: Iodine deficiency disorders; IQ: Intelligence quotient; USI: Universal salt iodization; WHO: World Health Organization; UIC: Urinary iodine concentration; IOM: United States Institute of Medicine; EAR: Estimated average requirement; AI: Adequate intake; RDA: Recommended daily allowance; RNI: Recommended nutrient intake; SCF: The European Union Scientific Committee on Foods; ND: Not determined

Author contribution CT, MP, and GP searched for bibliography; CT, $\mathrm{MP}$, and MB wrote the draft; $\mathrm{EN}, \mathrm{AO}, \mathrm{SM}$, and MB discussed, assembled, and revised the final version.

Funding Open access funding provided by Università degli Studi di Genova within the CRUI-CARE Agreement.

\section{Declarations}

Ethical approval, consent for publication, data availability Not applicable.

Conflict of interest The authors declare no competing interests.

Open Access This article is licensed under a Creative Commons Attribution 4.0 International License, which permits use, sharing, adaptation, distribution and reproduction in any medium or format, as long as you give appropriate credit to the original author(s) and the source, provide a link to the Creative Commons licence, and indicate if changes were made. The images or other third party material in this article are included in the article's Creative Commons licence, unless indicated otherwise in a credit line to the material. If material is not included in the article's Creative Commons licence and your intended use is not permitted by statutory regulation or exceeds the permitted use, you will need to obtain permission directly from the copyright holder. To view a copy of this licence, visit http://creativecommons.org/licenses/by/4.0/. 


\section{References}

1. Mcleod DSA, Cooper DS. The incidence and prevalence of thyroid autoimmunity. Endocrine. 2012;42:252-65.

2. Hayter SM, Cook MC. Autoimmunity reviews updated assessment of the prevalence, spectrum and case definition of autoimmune disease. Autoimmun Rev. 2012;11(10):754-65.

3. Giordano C, Richiusa P, Bagnasco M, Pizzolanti G, Di Blasi F, Sbriglia MS, et al. Differential regulation of Fas-mediated apoptosis in both thyrocyte and lymphocyte cellular compartments correlates with opposite phenotypic manifestations of autoimmune thyroid disease. Thyroid. 2001;11(3):233-44.

4. Caturegli P, De Remigis A, Rose NR. Hashimoto thyroiditis: clinical and diagnostic criteria. Autoimmun Rev. 2014;13(4-5):391-7.

5. Weetman A. Autoimmune thyroid disease. Endocrine. 2020;68(2):258-60.

6. Andersen S, Iversen F, Terpling S, Pedersen KM, Gustenhoff P, Laurberg P. Maturitas Iodine deficiency influences thyroid autoimmunity in old age - a comparative population-based study. Maturitas. 2012;71(1):39-43.

7. Prummel MF, Strieder T, Wiersinga WM. The environment and autoimmune thyroid diseases. Eur J Endocrinol. 2004;150(5):605-18.

8. Tomer Y. Mechanisms of autoimmune thyroid diseases: from genetics to epigenetics. Annu Rev Pathol. 2014;9:147-56.

9. Bagnasco M, Bossert I, Pesce G. Stress and autoimmune thyroid diseases. NeuroImmunoModulation. 2006;13(5-6):309-17.

10. Ferrari SM, Fallahi P, Antonelli A, Benvenga S. Environmental Issues in thyroid diseases. Front Endocrinol (Lausanne). 2017;8:50.

11. Pedersen IB, Knudsen N, Jørgensen T, Perrild H, Ovesen L, Laurberg P. Thyroid peroxidase and thyroglobulin autoantibodies in a large survey of populations with mild and moderate iodine deficiency. Clin Endocrinol (Oxf). 2003;58(1):36-42.

12. Vanderpump MP, Tunbridge WM, French JM, Appleton D, Bates $\mathrm{D}$, Clark F, et al. The incidence of thyroid disorders in the community: a twenty-year follow-up of the Whickham Survey. Clin Endocrinol (Oxf). 1995;43(1):55-68.

13. Hollowell JG, Staehling NW, Flanders WD, Hannon WH, Gunter EW, Spencer CA, et al. Serum TSH, T(4), and thyroid antibodies in the United States population (1988 to 1994): National Health and Nutrition Examination Survey (NHANES III). J Clin Endocrinol Metab. 2002;87(2):489-99.

14. Tozzoli R, Bagnasco M, Giavarina D, Bizzaro N. TSH receptor autoantibody immunoassay in patients with Graves ' disease: improvement of diagnostic accuracy over different generations of methods. Systematic review and meta-analysis. Autoimmun Rev. 2012;12(2):107-13.

15. Laurberg P. Iodine intake as a determinant of thyroid disorders in populations. Best Pract Res Clin Endocrinol Metab. 2010;24:13-27.

16. Dobson JE. The iodine factor in health and evolution. Geogr Rev. 2014;88(1):3-28.

17. Gorstein JL, Bagriansky J, Pearce EN, Kupka R, Zimmermann MB. Estimating the health and economic benefits of universal salt iodization programs to correct iodine deficiency disorders. Thyroid. 2020;30(12):1802-9.

18. Zimmermann MB, Boelaert K. Iodine deficiency and thyroid disorders. Lancet Diabetes Endocrinol. 2015;3(4):286-95.

19. Olivieri A, Trimarchi F, Vitti P. Global iodine nutrition 2020: Italy is an iodine sufficient country. J Endocrinol Invest. 2020;43(11):1671-2.
20. Iodine Global Network. Global Scorecard 2020. Available at site https://www.ign.org/cm_data/Global-Scorecard-2020-3-June2020.pdf. Accesed 23 April 2021.

21. Farebrother J, Zimmermann MB, Andersson M. Excess iodine intake: sources, assessment, and effects on thyroid function. Ann N Y Acad Sci. 2019;1446(1):44-65.

22. Rose NR, Rasooly L, Saboori AM, Burek CL. Linking iodine with autoimmune thyroiditis. Environ Health Perspect. 1999;107(Suppl 5):749-52.

23. Sundick RS, Bagchi N, Brown TR. The role of iodine in thyroid autoimmunity: from chickens to humans: a review. Autoimmunity. 1992;13(1):61-8.

24. Li M, Boyages SC. Iodide induced lymphocytic thyroiditis in the BB/W rat: evidence of direct toxic effects of iodide on thyroid subcellular structure. Autoimmunity. 1994;18(1):31-40.

25. Liu J, Mao C, Dong L, Kang P, Ding C. Excessive iodine promotes pyroptosis of thyroid follicular epithelial cells in Hashimoto's thyroiditis through the ROS-NF- $\kappa$ B-NLRP3 pathway. Front Endocrinol. 2019;10:1-10.

26. Di Dalmazi G, Hirshberg J, Lyle D, Freij JB, Caturegli P. Reactive oxygen species in organ-specific autoimmunity. Auto Immun Highlights. 2016;7(1):11-22.

27. Ruggeri RM, Campennİ A, Giuffrida G, Casciaro M, Barbalace MC, Hrelia S, et al. Oxidative stress as a key feature of autoimmune thyroiditis: an update. Minerva Endocrinol. 2020;45(4):326-44.

28. Ruggeri RM, Cristani MCBMT, Giovinazzo AAS, Trimarchi F. Serum levels of advanced glycation end products (AGEs) are increased and their soluble receptor (sRAGE) reduced in Hashimoto's thyroiditis. J Endocrinol Invest. 2020;43(9):1337-42.

29. Saboori AM, Rose NR, Burek CL. Iodination of human thyroglobulin (Tg) alters its immunoreactivity. II. Fine specificity of a monoclonal antibody that recognizes iodinated Tg. Clin Exp Immunol. 1998;113(2):303-8.

30. Dai YD, Rao VP, Carayanniotis G. Enhanced iodination of thyroglobulin facilitates processing and presentation of a cryptic pathogenic peptide. J Immunol. 2002;168(11):5907-11.

31. Burek CL, Talor MV. Environmental triggers of autoimmune thyroiditis. J Autoimmun. 2009;33(3-4):183-9.

32. Saboori AM, Rose NR, Bresler HS, Vladut-Talor M, Burek CL. Iodination of human thyroglobulin ( $\mathrm{Tg}$ ) alters its immunoreactivity. I. Iodination alters multiple epitopes of human $\mathrm{Tg}$. Clin Exp Immunol. 1998;113(2):297-302.

33. Rasooly L, Rose NR, Saboori AM, Ladenson PW, Burek CL. Iodine is essential for human $\mathrm{T}$ cell recognition of human thyroglobulin. Autoimmunity. 1998;27(4):213-9.

34. Weetman AP, McGregor AM. Autoimmune thyroid disease: further developments in our understanding. Endocr Rev. 1994;15(6):788-830.

35. Sercarz EE, Lehmann PV, Ametani A, Benichou G, Miller A, Moudgil K. Dominance and crypticity of T cell antigenic determinants. Annu Rev Immunol. 1993;11:729-66.

36. Carayanniotis G. The cryptic self in thyroid autoimmunity: The paradigm of thyroglobulin. Autoimmunity. 2003;36(6-7):423-8.

37. Latrofa F, Fiore E, Rago T, Antonangeli L, Montanelli L, Ricci $\mathrm{D}$, et al. Iodine contributes to thyroid autoimmunity in humans by unmasking a cryptic epitope on thyroglobulin. J Clin Endocrinol Metab. 2013;98(11):E1768-74.

38. Laurberg P, Pedersen KM, Hreidarsson A, Sigfusson N, Iversen E, Knudsen PR. Iodine intake and the pattern of thyroid disorders: a comparative epidemiological study of thyroid abnormalities in the elderly in Iceland and in Jutland. Denmark J Clin Endocrinol Metab. 1998;83(3):765-9. 
39. Suzuki H, Higuchi T, Sawa K, Ohtaki S, Horiuchi Y. "Endemic coast goitre" in Hokkaido. Japan Acta Endocrinol (Copenh). $1965 ; 50(2): 161-76$.

40. Leung AM, Braverman LE. Consequences of excess iodine. Nat Rev Endocrinol. 2014;10(3):136-42.

41. Markou K, Georgopoulos N, Kyriazopoulou V, Vagenakis AG. Iodine-induced hypothyroidism. Thyroid. 2001;11(5):501-10.

42. Laurberg P, Bülow Pedersen I, Knudsen N, Ovesen L, Andersen S. Environmental iodine intake affects the type of nonmalignant thyroid disease. Thyroid. 2001;11(5):457-69.

43. Stanbury JB, Ermans AE, Bourdoux P, Todd C, Oken E, Tonglet R, Vidor G, Braverman LE, Medeiros-Neto G. Iodine-induced hyperthyroidism: occurrence and epidemiology. Thyroid. 1998;8(1):83-100.

44. Petersen M, Knudsen N, Carlé A, Andersen S, Jørgensen T, Perrild $\mathrm{H}$, et al. Thyrotoxicosis after iodine fortification. a 21-year Danish population-based study. Clin Endocrinol. 2018;89(3):360-6.

45. Bürgi H. Iodine excess. Best Pract Res Clin Endocrinol Metab. 2010;24(1):107-15.

46. Bürgi H, Kohler M, Morselli B. Thyrotoxicosis incidence in Switzerland and benefit of improved iodine supply. Lancet. 1998;352(9133):1034.

47. Bülow Pedersen I, Laurberg P, Knudsen N, Jørgensen T, Perrild $\mathrm{H}$, Ovesen $\mathrm{L}$, et al. Increase in incidence of hyperthyroidism predominantly occurs in young people after iodine fortification of salt in Denmark. J Clin Endocrinol Metab. 2006;91(10):3830-4.

48. Vejbjerg P, Knudsen N, Perrild H, Carlé A, Laurberg P, Pedersen IB, et al. Effect of a mandatory iodization program on thyroid gland volume based on individuals' age, gender, and preceding severity of dietary iodine deficiency: a prospective, populationbased study. J Clin Endocrinol Metab. 2007;92(4):1397-401.

49. Krohn K, Führer D, Bayer Y, Eszlinger M, Brauer V, Neumann S, et al. Molecular pathogenesis of euthyroid and toxic multinodular goiter. Endocr Rev. 2005;26(4):504-24.

50. Kasagi K, Iwata M, Misaki T, Konishi J. Effect of iodine restriction on thyroid function in patients with primary hypothyroidism. Thyroid. 2003;13(6):561-7.

51. Yoon SJ, Choi SRKD, et al. The effect of iodine restriction on thyroid function in patients with hypothyroidism due to Hashimoto's thyroiditis. Yonsei Med J. 2003;44(2):227-35.

52. Yoshida H, Amino N, Yagawa K, Uemura K, Satoh M, Miyai K, et al. Association of serum antithyroid antibodies with lymphocytic infiltration of the thyroid gland: studies of seventy autopsied cases. J Clin Endocrinol Metab. 1978;46(6):859-62.

53. Arai T, Kurashima C, Utsuyama M, Sawabe M, Ito H. Measurement of anti-thyroglobulin and anti-thyroid peroxidase antibodies using highly sensitive radioimmunoassay: an effective method for detecting asymptomatic focal lymphocytic thyroiditis in the elderly. Endocr J. 2000;47(5):575-82.

54. Benvenga S, Trimarchi F. Changed presentation of Hashimoto's thyroiditis in North-Eastern Sicily and Calabria (Southern Italy) based on a 31-year experience. Thyroid. 2008;18(4):429-41.

55. Pedersen IB, Laurberg P. Chapter 60 - Antibodies to thyroid peroxidase and thyroglobulin in iodine deficiencies. In Preedy VR, Burrow GN, Watson RR eds. Comprehensive Handbook of Iodine, Academic Press, 2009, Pages 575-85.

56. Fenzi GF, Giani C, Ceccarelli P, Bartalena L, Macchia E, AghiniLombardi F, et al. Role of autoimmune and familial factors in goiter prevalence. Studies performed in a moderately endemic area. J Endocrinol Invest. 1986;9(2):161-4.

57. Loviselli A, Velluzzi F, Mossa P, Cambosu MA, Secci G, Atzeni F, et al. Sardinian Schoolchildren Study Group. The Sardinian Autoimmunity Study: 3. Studies on circulating antithyroid antibodies in Sardinian schoolchildren: relationship to goiter prevalence and thyroid function. Thyroid. 2001;11(9):849-57.
58. Sun J, Teng D, Li C, Peng S, Mao J, Wang W, et al. Association between iodine intake and thyroid autoantibodies: a cross-sectional study of 7073 early pregnant women in an iodine-adequate region. J Endocrinol Invest. 2020;43(1):43-51.

59. Levie D, Derakhshan A, Shu H, Broeren MAC, De Poortere RA. The association of maternal iodine status in early pregnancy with thyroid function in the SELMA study. Thyroid. 2019;29(11):1660-68.

60. Harach HR, Ceballos GA. Thyroid cancer, thyroiditis and dietary iodine: a review based on the Salta. Argentina model Endocr Pathol. 2008;19(4):209-20.

61. Kahaly GJ, Dienes HP, Beyer J, Hommel G. Iodide induces thyroid autoimmunity in patients with endemic goitre: a randomised, double-blind, placebo-controlled trial. Eur J Endocrinol. 1998;139(3):290-7.

62. Zimmermann MB, Moretti D, Chaouki N, Torresani T. Introduction of iodized salt to severely iodine-deficient children does not provoke thyroid autoimmunity: a one-year prospective trial in northern Morocco. Thyroid. 2003;13(2):199-203.

63. Mazziotti G, Premawardhana LD, Parkes AB, Adams H, Smyth PP, Smith DF, et al. Evolution of thyroid autoimmunity during iodine prophylaxis - the Sri Lankan experience. Eur J Endocrinol. 2003;149(2):103-10.

64. Giassa T, Mamali I, Gaki E, Kaltsas G, Kouraklis G, Markou KB, et al. Iodine intake and chronic autoimmune thyroiditis: a comparative study between coastal and mainland regions in Greece. Hormones (Athens). 2018;17(4):565-71.

65. Pedersen IB, Knudsen N, Carlé A, Vejbjerg P, Jørgensen T, Perrild $\mathrm{H}$, et al. A cautious iodization programme bringing iodine intake to a low recommended level is associated with an increase in the prevalence of thyroid autoantibodies in the population. Clin Endocrinol (Oxf). 2011;75(1):120-6.

66. Aghini Lombardi F, Fiore E, Tonacchera M, Antonangeli L, Rago $\mathrm{T}$, Frigeri M, et al. The effect of voluntary iodine prophylaxis in a small rural community : the pescopagano survey 15 years later. J Clin Endocrinol Metab. 2013;98:1031-9.

67. Hong A, Stokes B, Otahal P, Owens D, Burgess JR. Temporal trends in thyroid-stimulating hormone (TSH) and thyroid peroxidase antibody (ATPO) testing across two phases of iodine fortification in Tasmania (1995-2013). Clin Endocrinol (Oxf). 2017;87(4):386-93.

68. Khattak RM, Ittermann T, Nauck M, Below H, Völzke H. Monitoring the prevalence of thyroid disorders in the adult population of Northeast Germany. Popul Health Metr. 2016;14:39 e-collection.

69. Wan S, Jin B, Ren B, Qu M, Wu H, Liu L, et al. The relationship between high iodine consumption and levels of autoimmune thyroiditis-related biomarkers in a Chinese population: a metaanalysis. Biol Trace Elem Res. 2020;196(2):410-8.

70. Bournaud C, Orgiazzi JJ. Iodine excess and thyroid autoimmunity. J Endocrinol Invest. 2003;26(2 Suppl):49-56.

71. Wang B, He W, Li Q, et al. U-shaped relationship between iodine status and thyroid autoimmunity risk in adults. Eur J Endocrinol. 2019;181(3):255-266.

72. Teng X, Shan Z, Chen Y, et al. More than adequate iodine intake may increase subclinical hypothyroidism and autoimmune thyroiditis: a cross-sectional study based on two Chinese communities with different iodine intake levels. Eur J Endocrinol. 2011;164(6):943-950.

73. Miranda DM, Massom JN, Catarino RM, Santos RT, Toyoda SS, Marone MM, et al. Impact of nutritional iodine optimization on rates of thyroid hypoechogenicity and autoimmune thyroiditis: a cross-sectional, comparative study. Thyroid. 2015;25(1):118-24.

74. Teng D, Yang W, Shi X, Li Y, Ba J, Chen B, et al. An inverse relationship between iodine intake and thyroid antibodies: a 
national cross-sectional survey in Mainland China. Thyroid. 2020;30(11):1656-65.

75. Duntas LH. The catalytic role of iodine excess in loss of homeostasis in autoimmune thyroiditis. Curr Opin Endocrinol Diabetes Obes. 2018;25(5):347-35.

76. Li Y, Teng D, Shan Z, et al. Antithyroperoxidase and antithyroglobulin antibodies in a five-year follow-up survey of populations with different iodine intakes. J Clin Endocrinol Metab. 2008;93:1751-7.

77. Petersen M, Bülow Pedersen I, Knudsen N, Andersen S, Jørgensen $\mathrm{T}$, Perrild $\mathrm{H}$, et al. Changes in subtypes of overt thyrotoxicosis and hypothyroidism following iodine fortification. Clin Endocrinol (Oxf). 2019;91(5):652-9.

78. Jayatissa R, Okosieme OE, Ranasinghe S, et al. Thyroid autoimmunity and dysfunction in sri lankan children and adolescents after 22 years of sustained universal salt iodization. Thyroid. 2021. https://doi.org/10.1089/thy.2020.0798

79. Biondi B, Cooper DS. The clinical significance of subclinical thyroid dysfunction. Endocr Rev. 2008;29(1):76-131.

80. Rago T, Chiovato L, Grasso L, Pinchera A, Vitti P. Thyroid ultrasonography as a tool for detecting thyroid autoimmune diseases and predicting thyroid dysfunction in apparently healthy subjects. J Endocrinol Invest. 2001;24(10):763-769.

81. Cantisani TRV, Chiovato FIL, Durante RGC, Spiezia AFS. Thyroid ultrasonography reporting : consensus of Italian Thyroid Association (AIT), Italian Society of Endocrinology (SIE), Italian
Society of Ultrasonography in Medicine and Biology (SIUMB) and Ultrasound Chapter of Italian Society of Medical Radiology (SIRM). J Endocrinol Invest. 2018;41(12):1435-43.

82. De Angelis S, Bagnasco M, Moleti M, Regalbuto C, Tonacchera $\mathrm{M}$, Vermiglio $\mathrm{F}$, et al. Obesity and Monitoring iodine nutritional status in schoolchildren: is body mass index a factor to consider? Thyroid. 2021. https://doi.org/10.1089/thy.2020.0189

83. Agmon-levin N, Lian Z, Shoenfeld Y. Explosion of autoimmune diseases and the mosaic of old and novel factors. Cell Mol Immunol. 2011;8(3):189-92.

84. Rose NR. Prediction and prevention of autoimmune disease in the 21st century: a review and preview. Am J Epidemiol. 2016;183(5):403-6.

85. Rook GAW. Hygiene hypothesis and autoimmune diseases. Clin Rev Allergy Immunol. 2012;42(1):5-15.

86. Kondrashova A, Viskari H, Haapala A, et al. Serological Evidence of thyroid autoimmunity among schoolchildren in two different socioeconomic environments. J Clin Endocrinol Metab. 2008;93:729-34.

Publisher's note Springer Nature remains neutral with regard to jurisdictional claims in published maps and institutional affiliations. 